\title{
Heat loads in inboard limited L-mode plasmas in TCV
}

\author{
F. Nespoli ${ }^{*, 1}$, B. Labit, I. Furno, G.P. Canal, A. Fasoli, the TCV team \\ Ecole Polytechnique Fédérale de Lausanne, Centre de Recherches en Physique des Plasmas, 1015 Lausanne, Switzerland
}

\section{A R T I C L E I N F O}

Article history:

Available online $\mathrm{xxxx}$

\begin{abstract}
A B S T R A C T
Infrared thermography is used in TCV to measure the heat flux deposited onto the graphite tiles of the inner wall. The heat flux radial profile is found to be well described by the sum of a main parallel component and a non negligible cross-field component. The latter accounts for about $20 \%$ of the deposited heat flux. The parallel component shows an enhancement around the contact point in all discharges under consideration. Main plasma parameters, such as density, current, elongation and triangularity have been varied, allowing for empirical scalings of the heat fluxes.
\end{abstract}

(c) 2015 Elsevier B.V. All rights reserved.

\section{Introduction}

In the scrape-off layer (SOL) of a tokamak, most of the losses from the last closed flux surface (LCFS) are believed to occur at the outer mid plane (OMP). The heat flux along the field lines is usually assumed to decay exponentially with the distance from the LCFS at the OMP, $r_{u}$, as $q_{\|}=q_{\| 0} \exp \left(-r_{u} / \lambda_{q}\right)$ where $q_{\| 0}$ is the flux at the separatrix and $\lambda_{q}$ is the heat flux decay length in the SOL. The heat flux deposited on the inboard limiter is $q_{\text {dep }}=q_{\|} \sin \alpha$, where $\alpha$ is the angle between the magnetic field and the direction tangent to the solid surface.

Inboard limited discharges in JET recently have shown an enhanced flux at the limiter up to a factor 3 with respect to what is foreseen from the heat flux measured at the OMP [1]. In these discharges, $q_{\|}$is actually better described by a double exponential

$q_{\|}=q_{\| s} \exp \left(-r_{u} / \lambda_{s}\right) \Theta\left(r_{0}-r_{u}\right)+q_{|| l} \exp \left(-r_{u} / \lambda_{l}\right) \Theta\left(r_{u}-r_{0}\right)$,

where $\lambda_{s}<\lambda_{l}, r_{0}$ corresponds to the radial position where the slope of the profile changes, and $\Theta(x)$ is the Heaviside function. Inboard limited L-mode plasmas are foreseen for ITER startup and ramp down phases [2]. If such an enhanced heat flux is present, the heat load on the limiter could result in the melting of the beryllium first wall panels, according to their present design [3].

A series of dedicated experiments is performed on TCV to investigate the presence of such an enhanced heat flux. In TCV, the inboard limiter is formed by 32 graphite tiles, shaped in order to minimize the heat loads [4]. These tiles constitute a

* Corresponding author at: Ecole Polytechnique Fédérale de Lausanne, Centre de Recherches en Physique des Plasmas, Station 13, 1015 Lausanne, Switzerland.

E-mail address: federico.nespoli@epfl.ch (F. Nespoli).

1 Presenting author. quasi-continuous toroidal limiter, similar to the ITER first wall. $\mathrm{TCV}$ is a unique device for studying the physics of plasma-wall interactions, because of the limiter configuration and the extreme flexibility for plasma shaping.

\section{Experimental setup and measurements}

The experimental setup is shown in Fig. 1. The main diagnostic used in this series of experiments is a fast framing infrared (IR) camera monitoring the temperature of the graphite tiles of the central column. The camera is equipped by a CdHgTe detector ( $256 \times 256$ pixels), sensitive to radiation in the wavelength range from 1.5 to $5.1 \mu \mathrm{m}$. Nevertheless, for some discharges, a band pass filter $(4.5-5.1 \mu \mathrm{m})$ was part of the optics, which might affect the analysis. The field of view (FOV), shown in Fig. 1 by the blue dashed lines, covers approximately 3 tiles in the vertical direction and 3 tiles in the toroidal direction. The FOV determines the portion of the SOL which can be investigated, in particular it defines $r_{u \text { max }}$.

Moreover, ten thermocouples (TC), embedded in the central column tiles, are monitoring their temperature $(1.66 \mathrm{~Hz}$ acquisition frequency) and are providing a measurement of the energy deposited on the inner wall during the plasma discharge. In addition to the IR, the central column is equipped with an array of flushmounted Langmuir Probes (LPs), providing an additional measurement of the deposited heat flux defined as $q_{\mathrm{dep}, \mathrm{LP}}=\gamma_{\text {sheath }} J_{\mathrm{sat}} T_{e}$. However, the small angle of incidence of the magnetic field at the probe locations $\left(\leqslant 2^{\circ}\right)$ complicates the interpretation of the LP data. The comparison with the IR data is therefore delicate. Finally, edge electron density and temperature are provided by Thomson scattering measurements.

In these experiments, inboard limited L-mode plasmas are investigated with ohmic heating only. 


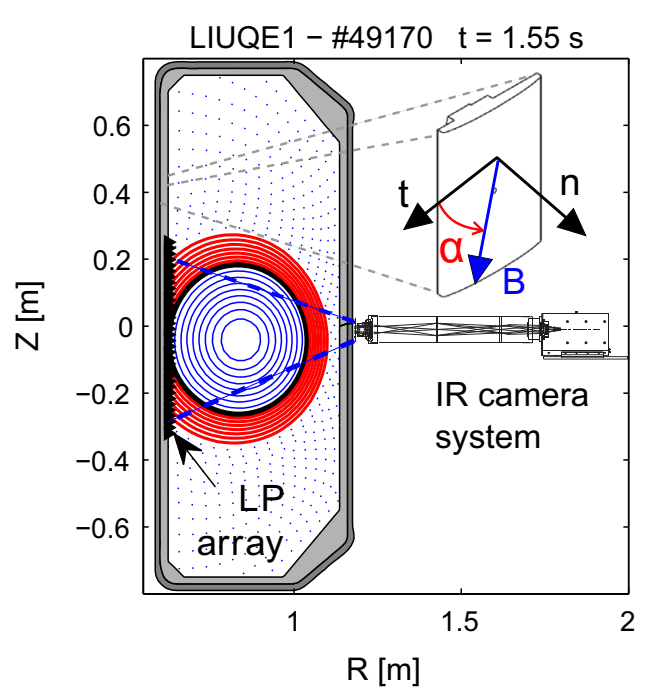

Fig. 1. Schematics of the diagnostic setup showing the fast framing IR camera with its optics and its field of view (blue dashed line), the LP array (black triangles), the LCFS (black thick line) and the portion of the SOL under investigation (red continuous lines). The geometry of the angle $\alpha$ between the direction tangent to the graphite tile surface and the magnetic field line $\mathbf{B}$ is also shown. (For interpretation of the references to color in this figure legend, the reader is referred to the web version of this article.)

The dataset consists of 35 steady-state phases among which some plasma parameters have been varied: the plasma current $140 \leqslant I_{p}[\mathrm{kA}] \leq 225$, the plasma density $1.7 \leqslant n_{e, \mathrm{av}}\left[10^{19} \mathrm{~m}^{-3}\right] \leqslant 6$, the plasma elongation $\kappa=1,1.2,1.4$ and triangularity $\delta=-0.4$, $-0.2,0,0.2,0.4$.

\section{IR data analysis method}

IR images are converted to temperature profiles using an in situ calibration. A heated graphite tile is used for the calibration and to focus the optics. Each IR image is corrected to compensate for non uniformities associated with optical aberration of the optical system. The temperature images are then mapped onto the real space, accounting for perspective deformations, resulting in a twodimensional (2D) temperature profile $T(z, R \phi, t)$ which is consistent with the tiles shape and their mutual shadowing (Fig. 2), and with the temperature range measured by the TC.

The THEODOR code [5], which solves the heat diffusion equation, is used to compute the heat flux deposited onto the tiles. Since the tile thickness is not uniform on the ion drift side and some tiles are misaligned in that region, only the electron drift side of the limiter is considered. The analysis is thus restricted to one tile at $z=0$ and half of the tile above this one (Fig. 2). This selected region is divided into 50 horizontal slices and for each slice THEODOR is run. Assuming that the heat diffuses mainly in the depth of the tile, the 2D map of the deposited heat $q_{\mathrm{dep}}(z, R \phi, t)$ can be reconstructed, as shown in Fig. 3a. Two THEODOR input parameters, the tile thickness $d_{\text {tile }}$ and the emissivity of the top layer of the tile $\alpha_{\text {top }}$, are adjusted in order to cancel the heat flux on the tile after the end of the discharge.

The level of heat flux computed from the LPs using $\gamma_{\text {sheath }}=5$ [6] is comparable to the one measured from the IR, once a background is subtracted. In the surrounding of the contact point, where $\alpha \sim 0$ (grazing incidence), a lower $\gamma_{\text {sheath }}$ would be needed for a better agreement. The deposited energy computed from the TC is in good agreement with the one from the IR, evaluated as $E_{\text {dep }}=\iiint q_{\text {dep }} d z d R \phi d t$.

Using the magnetic equilibrium reconstructed with the LIUQE code [7], each point of the tile surface is mapped from the real space coordinates $(z, R \phi)$ to the coordinates $\left(r_{u}, \alpha\right)$, as shown in

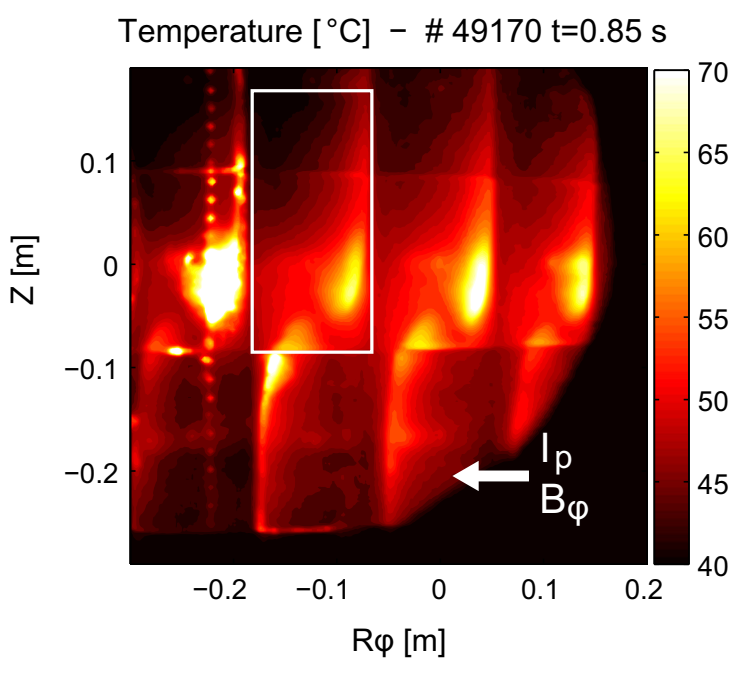

Fig. 2. 2D image of the temperature after calibration and optical corrections. LPs are visible on the left of the image. The white box indicates the restricted area for the analysis. The differences between the temperature of different tiles are consistent with their different thickness. The direction of the toroidal magnetic field and plasma current is displayed with an arrow.

Fig. 3b and c, where $r_{u}$ is the distance from the LCFS at the midplane and $\alpha$ is the angle between the magnetic field and the direction tangent to the solid surface.

The heat flux $q_{\text {dep }}$ is time-averaged over the duration of each steady-state phase $(\sim 0.5 \mathrm{~s})$, the standard deviation providing an estimate for the statistical error in the computed $q_{\mathrm{dep}}$. Finally, only the points lying between the line $\alpha=0$ (black dots Fig. $3 b$ and $c$ ) and the line providing the maximum of $q_{\text {dep }}$ for each slice (black crosses) are kept. We exclude the shadowed regions from the analysis, as well as the region around the bright spot in the middle of the tile visible in Fig. 3a, due to the supporting screw.

\section{Results}

The measured heat flux deposited on the tile $q_{\text {dep }}$ is modeled as $q_{\mathrm{dep}}\left(r_{u}, \alpha\right)=q_{\|}\left(r_{u}\right) \sin \alpha+q_{\perp}\left(r_{u}\right) \cos \alpha+q_{\mathrm{BG}}$,

where $q_{\|}$and $q_{\perp}$ are the parallel and cross-field components of the heat flux respectively. The background heat flux $q_{\mathrm{BG}}$ results partly from IR reflections and partly from heating of the tile by radiation from the plasma and energetic neutrals. From Eq. (2), at $\alpha=0$ only $q_{\perp}$ and $q_{\mathrm{BG}}$ contribute to the deposited heat flux. Conversely to some other tokamaks first wall, where the angle of incidence $\alpha$ is usually larger than a couple of degrees, the limiter geometry with $\alpha=0$ on a portion of the tile allows for a correct estimate of the perpendicular component of the heat flux. The latter is non negligible here, being $q_{\perp}$ at the contact point around $20 \%$ of the maximum of $q_{\text {dep }}$ on the tile. Assuming that the perpendicular heat flux can be described by $q_{\perp}\left(r_{u}\right)=q_{\perp, 0} \exp \left(-r_{u} / \lambda_{\perp}\right)$, a least-square fit of $q_{\text {dep }}\left(r_{u}, \alpha=0\right)$ (black line, Fig. 4 a) is performed to estimate $q_{\perp 0}, \lambda_{\perp}$ and $q_{\mathrm{BG}}$. For the entire database, the decay length $\lambda_{\perp}$ is found to vary from $1.5 \mathrm{~mm}$ to $7.6 \mathrm{~mm}$; however, no satisfactory scaling with plasma parameters could be found. Conversely, a nonlinear regression provides an empirical scaling (Fig. 5) for $q_{\perp, 0}$ that reads

$q_{\perp, 0\left[\mathrm{~kW} / \mathrm{m}^{2}\right]}=1.99 \cdot 10^{4} I_{p[\mathrm{MA}]}^{2.51} \kappa^{-0.92} n_{e, \mathrm{av}\left[10^{-0.9} \mathrm{~m}^{-3}\right]}^{-{ }^{-0.1}}|\delta|^{0.13}$.

The main trend is the increase of $q_{\perp, 0}$ with the plasma current, consistent with an increase of the ohmic power. Having determined the perpendicular and background heat flux, we can compute the parallel heat flux from Eq. (2) as: 

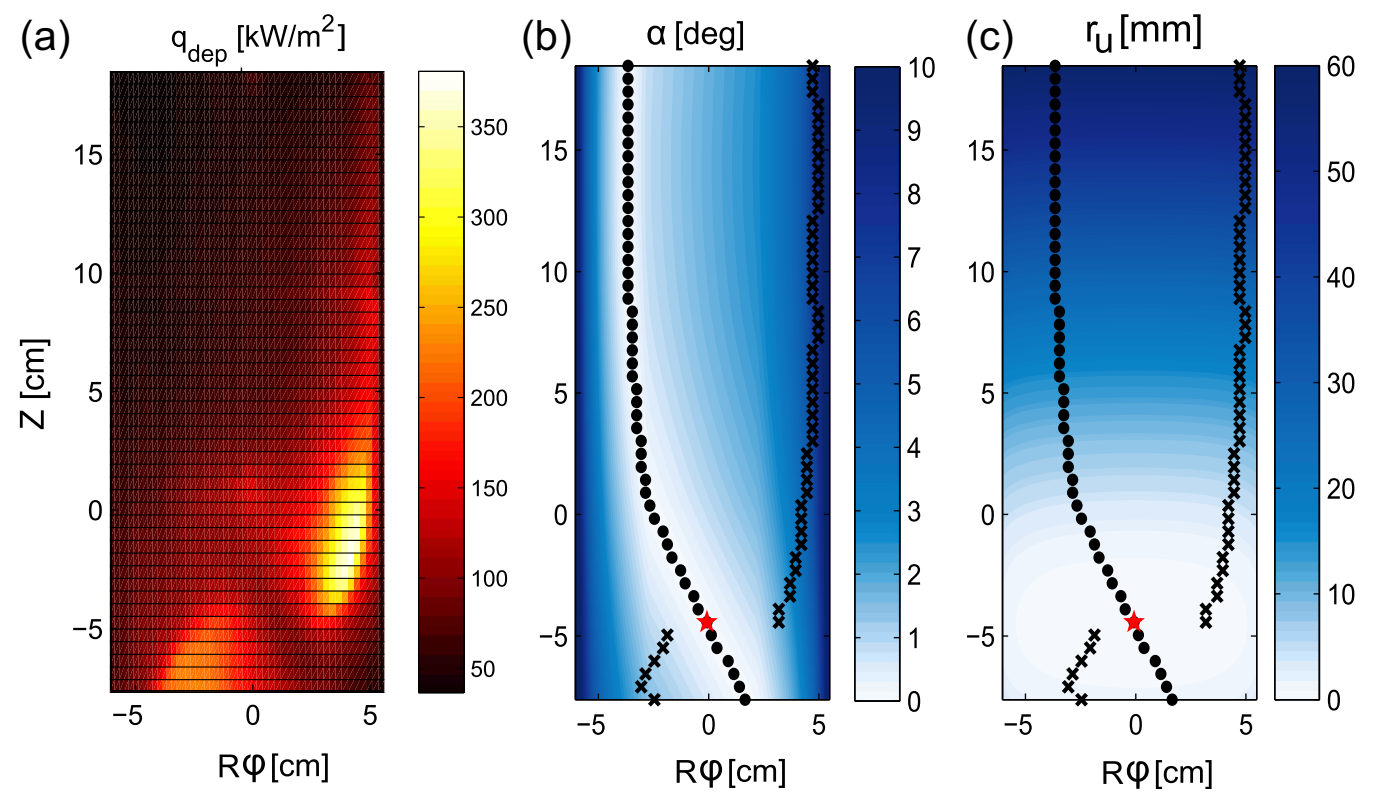

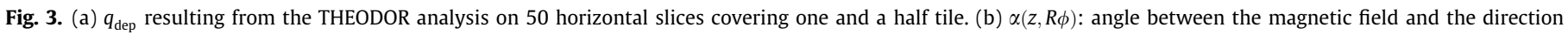

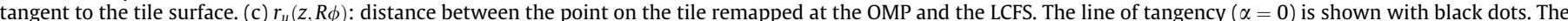

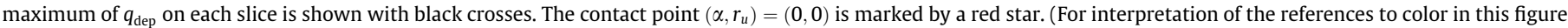
legend, the reader is referred to the web version of this article.)

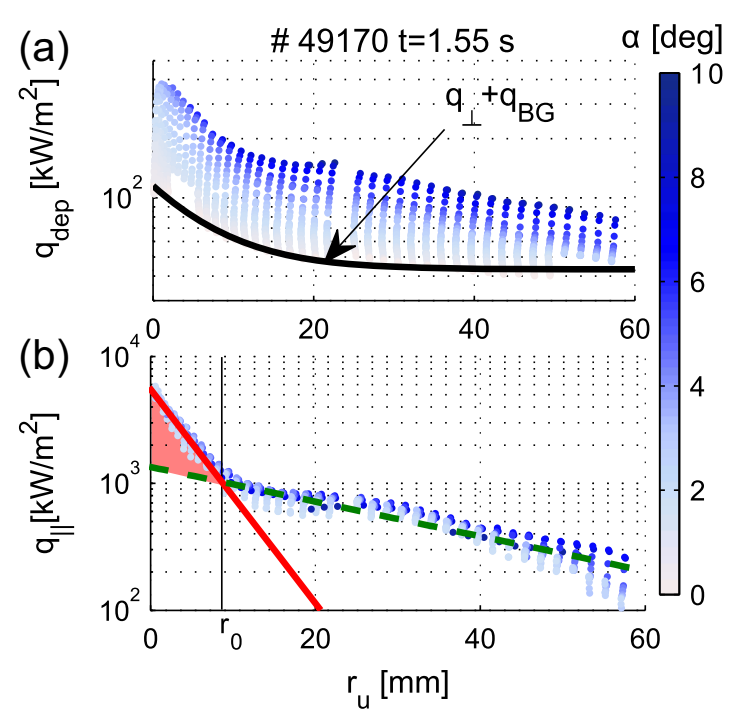

Fig. 4. (a) Deposited heat $q_{\text {dep }}\left(r_{u}\right)$ color-coded with the angle of incidence $\alpha$. Fitting the black line gives the perpendicular heat flux and the background flux. (b) Parallel heat flux for $\alpha \geqslant 2^{\circ}$ fitted with 2 exponentials with $\lambda_{s}=5.2 \mathrm{~mm}$ (continuous red line) and $\lambda_{l}=33.8 \mathrm{~mm}$ (dashed green line), and excess heat in the SOL given by Eq. (5) (shaded area). (For interpretation of the references to color in this figure legend, the reader is referred to the web version of this article.)

$q_{\|}\left(r_{u}\right)=\frac{1}{\sin \alpha}\left[q_{\mathrm{dep}}\left(r_{u}, \alpha\right)-q_{\perp}\left(r_{u}\right) \cos \alpha-q_{\mathrm{BG}}\right]$.

This is plotted in Fig. $4 \mathrm{~b}$ for $\alpha \geqslant 2^{\circ}$ and clearly shows the presence of two scale lengths, such that $q_{\|}$cannot be fitted with a single exponential. Indeed, the parallel heat flux is well described by two exponentials with different decay lengths according to Eq. (1), similarly to JET results. This is observed for all the analyzed discharges, independently of the heating power or the plasma shaping. A leastsquare fit of the data with Eq. (1) provides the parameters $q_{s}, q_{l}, \lambda_{s}, \lambda_{l}$ and $r_{0}$. Over the entire database, we find that the short decay length $\lambda_{s}$ is of the same order as $\lambda_{\perp}: 1.6 \mathrm{~mm}<\lambda_{s}<5.8 \mathrm{~mm}$,

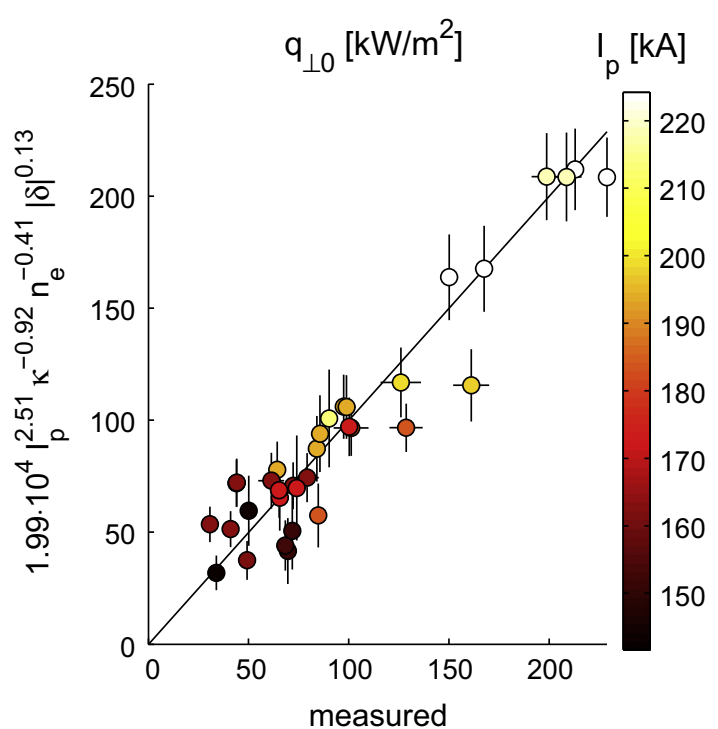

Fig. 5. Empirical scaling for the heat flux at the contact point $q_{\perp 0}$, color coded with the plasma current $I_{p}$. (For interpretation of the references to color in this figure legend, the reader is referred to the web version of this article.)

while the long decay length $\lambda_{l}$ extends from $11.9 \mathrm{~mm}$ to $31.8 \mathrm{~mm}$. The average values over the entire database are $\bar{\lambda}_{s}=3.3 \pm 1.0 \mathrm{~mm}$ and $\bar{\lambda}_{l}=17.7 \pm 5.0 \mathrm{~mm}$, respectively.

In the following, we compare the experimental scale lengths with recent theoretical and heuristic scalings. Based on numerical simulations and the gradient removal theory [8], a scaling for the pressure scale length $L_{P}$ for limited circular plasmas predicts that $L_{P}$ depends strongly on the plasma current $\left(\propto I_{p}^{-8 / 7}\right)$ [9]. Noting that $\lambda_{q}=L_{P} / C$ with $C=\frac{\eta+3 / 2}{\eta+1}$ and $\eta=L_{T} / L_{n}$, we compute $\lambda_{q}$ assuming $\eta=0.8$ to compare with the experimentally measured $\lambda_{s}$ and $\lambda_{l}$ (Fig. 6a). This scaling is found to predict the right order of magnitude for the long decay length $\lambda_{l}$ and so not for $\lambda_{s}$. Two additional 

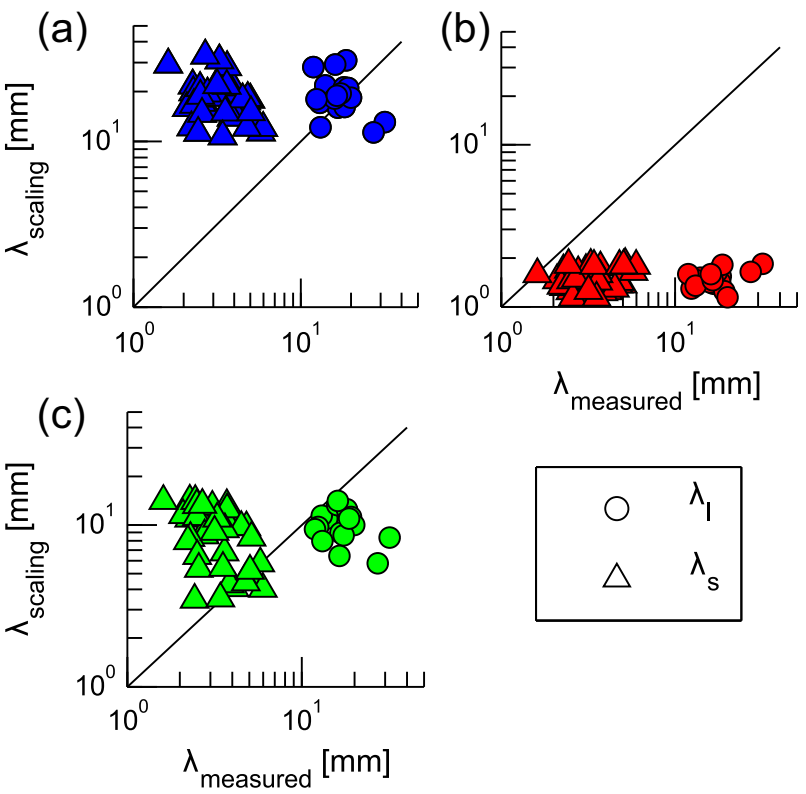

Fig. 6. Comparison between the measured decay lengths $\lambda_{s}$ (triangles) and $\lambda_{1}$ (circles) with the scaling from [9] (a), the heuristic drift-based scaling [10] (b) and the ITER database scaling [11] (c).

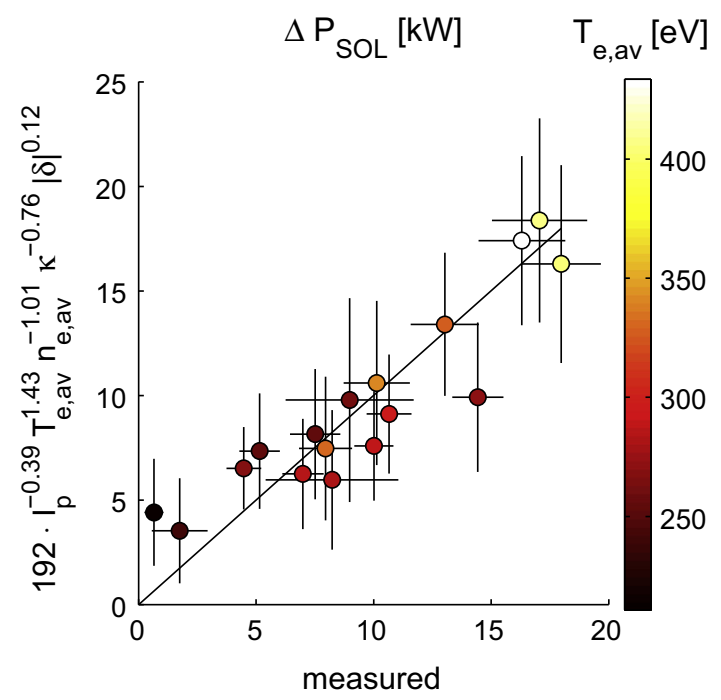

Fig. 7. Empirical scaling for the excess power in the SOL $\Delta P_{\text {SOL }}$, color coded with the volume averaged electron temperature $T_{e, \mathrm{av}}$. (For interpretation of the references to color in this figure legend, the reader is referred to the web version of this article.)

scalings developed for diverted plasmas have been tested, namely the one derived from the heuristic drift (HD) model [10] and the empirical one from the ITER database [11]. The former predicts $\lambda_{q} \propto I_{p}^{-9 / 8}\left(1+\kappa^{2}\right)^{5 / 8}$ (Fig. 6b) while the latter yields $\lambda_{q} \propto q_{95}^{0.5} n_{e, \mathrm{av}, 19}^{0.9}$ (Fig. 6c). Both scalings tend to better predict $\lambda_{s}$ then $\lambda_{l}$. Furthermore, a least-square fit of the same data presented here with a sum of two exponentials instead of Eq. (1), would produce an even shorter $\lambda_{s}$, leading to a better agreement with the HD model [12].
The observed enhanced heat load in the vicinity of the contact point could be a problem for ITER if it is too large. To be more quantitative, we define the excess of power that enters in the SOL due to the narrow feature as

$\Delta P_{\mathrm{SOL}}=4 \pi R_{\mathrm{LCFS}} \frac{B_{\theta}}{B_{\phi}} \int_{0}^{r_{0}}\left(q_{s} e^{-r_{u} / \lambda_{s}}-q_{l} e^{-r_{u} / \lambda_{l}}\right) d r_{u}$.

This is represented by the shaded area in Fig. $4 \mathrm{~b}$. A nonlinear regression provides an empirical scaling for $\Delta P_{\mathrm{SOL}}$, shown in Fig. 7

$\Delta P_{\mathrm{SOL}[\mathrm{kW}]}=192 T_{e, \mathrm{av}[\mathrm{keV}]}^{1.43} n_{e, \mathrm{av}\left[10^{19} \mathrm{~m}^{-3}\right]}^{-1.0{ }_{p}[\mathrm{MA}]}{ }^{-0.39} \kappa^{-0.76}|\delta|^{0.12}$.

The excess power in the SOL is therefore found to increase mainly with the electron temperature, and to decrease with plasma density, current and elongation.

\section{Conclusions}

Dedicated experiments were performed in TCV in order to investigate the enhancement of the heat deposition onto the limiter close to the contact point. For all the discharges, the parallel heat flux profile exhibits a double decay length in the SOL, with a short decay length around $3 \mathrm{~mm}$ and a longer decay length around $18 \mathrm{~mm}$. The dependence of the heat flux on the plasma parameters has been investigated. Together with other dedicated experiments on various tokamaks (JET [1], DIII-D [13], COMPASS [14]) requested by the ITER Organization, these results led to a change in the ITER first wall design [15], in order to handle a heat flux with a radial profile featuring an additional (shorter) decay length.

Further experiments including measurements with a reciprocating LP are foreseen in TCV to complement the present results with heat flux radial profiles measured at the OMP. Dedicated numerical simulations with the GBS [16] and EMC3-Eirene [17] codes are foreseen to have a better understanding of the heat flux enhancement at the limiter.

\section{Acknowledgments}

The authors wish to thank H. Reimerdes, R.A. Pitts, M. Kocan, R. Goldston, P. Stangeby and J. Horacek for useful discussions. FN thanks A. Hermann for his help about the THEODOR code. This work is partly funded by the Fonds National Suisse de la Recherche Scientifique and by EURATOM. The views and opinions expressed herein do not necessarily reflect those of the European Commission.

\section{References}

[1] G. Arnoux et al., Nucl. Fusion 53 (2013) 073016.

[2] R.A. Pitts et al., J. Nucl. Mater. 415 (2011) 957-964.

[3] A.R. Raffray et al., Nucl. Fusion 54 (2014).

[4] R.A. Pitts et al., Nucl. Fusion 39 (1999)

[5] A. Herrmann, ECA 25A (2001) 2109-2112.

[6] H. Reimerdes et al., Plasma Phys. Controlled Fusion 55 (2013) 124027.

[7] F. Hofmann, G. Tonetti, Nucl. Fusion 28 (1988) 519.

[8] P. Ricci, B.N. Rogers, Phys. Plasmas 20 (2013) 010702.

[9] F.D. Halpern et al., Nucl. Fusion 53 (2013) 122001.

[10] R.J. Goldston, Nucl. Fusion 52 (2012) 013009.

[11] ITER Physics Basis, Nucl. Fusion 39 (1999) 2391.

[12] R.J. Goldston, http://dx.doi.org/10.1016/j.jnucmat.2014.10.080.

[13] P.C. Stangeby et al., http://dx.doi.org/10.1016/j.jnucmat.2014.09.051.

[14] J. Horacek et al., http://dx.doi.org/10.1016/j.jnucmat.2014.11.132.

[15] M. Kocan, R.A. Pitts, et al., Nucl. Fusion, 2014 (submitted for publication).

[16] P. Ricci et al., Plasma Phys. Controlled Fusion 54 (2012) 124047.

[17] Y. Feng et al., Contrib. Plasma Phys. 44 (2004) 57. 